\title{
Long-time behavior of global weak solutions for a Beris-Edwards type model of nematic liquid crystals
}

\author{
Blanca CLIMENT-EZQUERRA ${ }^{1}$ and Francisco Guillen-Gonzalez ${ }^{1}$ \\ ${ }^{1}$ Universidad de Sevilla
}

November 16, 2020

\begin{abstract}
We consider a generalization of the standard Beris-Edwards system modeling incompressible liquid crystal flows of nematic type. This couples a Navier-Stokes system for the fluid velocity with an evolution equation for the Q-tensors variable describing the direction of liquid crystal molecules. The convergence at infinite time for global solutions is studied and we prove that whole trajectory goes to a single equilibrium by using a Lojasiewicz-Simon's result.
\end{abstract}

\section{Hosted file}

NewQtensorBigT.pdf available at https://authorea.com/users/376331/articles/493313-1ongtime-behavior-of-global-weak-solutions-for-a-beris-edwards-type-model-of-nematic-liquidcrystals 